\title{
Bone morphology of the femur and tibia captured by statistical shape modelling predicts rapid bone loss in acute spinal cord injury patients.
}

\section{Authors:}

Delaram Varzi ${ }^{\mathrm{a}}$, Sylvie AF Coupaud ${ }^{\mathrm{b}, \mathrm{c}}$, Mariel Purcell ${ }^{\mathrm{c}}$, David B Allan ${ }^{\mathrm{c}}$, Jennifer S Gregory ${ }^{\mathrm{a}}$, Rebecca J Barr ${ }^{\mathrm{a}}$

\begin{abstract}
Affiliations:
${ }^{\mathrm{a}}$ Musculoskeletal Research Programme, University of Aberdeen, Aberdeen, UK, ${ }^{\mathrm{b}}$ Department of Biomedical Engineering, University of Strathclyde, Glasgow, UK, ${ }^{\mathrm{c}}$ Scottish Centre for Innovation in Spinal Cord Injury, Queen Elizabeth National Spinal Injuries Unit, Southern General Hospital, Glasgow, UK.
\end{abstract}

\section{Corresponding Author:}

Dr Rebecca Barr

Postal address:

Rm 2.23 IMS

Foresterhill

Aberdeen AB25 2ZD

E-mail:r.j.barr@abdn.ac.uk

Telephone number: +44 (0)1224 438062 


\section{Abstract}

After spinal cord injury (SCI), bone loss in the paralysed limbs progresses at variable rates. Decreases in bone mineral density (BMD) in the first year range from $1 \%$ (slow) to $40 \%$ (rapid). In chronic SCI, fragility fractures commonly occur around the knee, with significant associated morbidity. Osteoporosis treatments await full evaluation in SCI, but should be initiated early and targeted towards patients exhibiting rapid bone loss. The potential to predict rapid bone loss from a single bone scan within weeks of a SCI was investigated using Statistical Shape Modelling (SSM) of bone morphology, hypothesis: baseline bone shape predicts bone loss at 12-months post-injury at fracture-prone sites.

In this retrospective cohort study 25 SCI patients (median age, 33 years) were scanned at the distal femur and proximal tibia using peripheral Quantitative Computed Tomography at $<5$ weeks (baseline), 4, 8 and 12 months post-injury. An SSM was made for each bone. Links between the baseline shape-modes and 12-month total and trabecular BMD loss were analysed using multiple linear regression.

One mode from each SSM significantly predicted bone loss (age-adjusted $\mathrm{P}<0.05 \mathrm{R}^{2}=0.37$ 0.61 ) at baseline. An elongated intercondylar femoral notch (femur mode $4,+1 \mathrm{SD}$ from the mean) was associated with $8.2 \%$ additional loss of femoral trabecular BMD at 12-months. A more concave posterior tibial fossa (tibia mode $3,+1 \mathrm{SD}$ ) was associated with $9.4 \%$ additional 12-month tibial trabecular BMD loss.

Baseline bone shape determined from a single bone scan is a valid imaging biomarker for the prediction of 12-month bone loss in SCI patients. 


\section{Keywords:}

Spinal cord injuries, Osteoporosis, Tibia, Femur, statistical shape model, pQCT 


\section{Introduction}

Every year 130,000 people worldwide, survive a traumatic Spinal Cord Injury (SCI)[1]. In developed economies these patients can now expect near-normal lifespans, but have to face the many complications of SCI including osteoporosis and osteoporotic fractures[2-4]. Osteoporotic fractures occur in men and women, young and old; they are frequently lowenergy, occurring during transfer activities, such as from wheelchair to bed or bed to chair, during rehabilitation activities or simply as a result of turning in bed[4] and are at least twice as common as in the general population[5]. The distal femur and proximal tibia are the commonest sites of bone loss and fractures.

At first glance the implications of lower limb fractures in patients with SCI may seem insignificant as patients are already non-ambulatory. However, fractures can have serious consequences with morbidity exceeding non-SCI fracture populations[6] often compounded by a delay in recognising the fracture. SCI related lower limb fractures are difficult to treat as the fractured bone is commonly fragile and porous making surgical fixation complicated, whilst the other standard option, plaster casts can cause pressure ulcers in the limb they are applied to. Other complications include mal-union and deformity (another cause of pressure ulcers), extended periods of bed rest, prolonged hospitalisation and decreased independence[7].

\section{Abbreviations}

Spinal Cord Injury (SCI), Statistical Shape Modelling (SSM), Queen Elizabeth National Spinal Injury Unit (QENSIU), American Spinal Injury Association (ASIA), ASIA Impairment Score (AIS) 
No guidelines exist for the treatment of osteoporosis in SCI patients, and clinicians generally take a reactive rather than a preventative approach to treating this group. Guidelines for postmenopausal osteoporosis[8, 9], have limited value for treating SCI osteoporosis because of differences in the pathophysiology and co-morbidities[2-4]. Furthermore, there is no clear equivalent of FRAX for fracture risk calculation for SCI patients[10]. Current available treatments are often intensive, life-long and have undesirable side effects[11]. Importantly, many of the treatments have either only worked, or worked best, when administered in the acute stage after injury[12]. All these challenges that patients and clinicians face could be circumvented by establishing a preventative target to treat approach. Identifying those at risk of rapid bone loss would help greatly in initiating therapy in the early stage after injury, when it is most likely to be effective[12, 13].

The majority of acute bone loss occurs in the first 1-2 years after injury[14, 15]. Bone Mineral Density (BMD) gradually levels off at around 3-7 years post-injury with variations in the rate depending on the anatomical location[16]. SCI osteoporosis is "sublesional" i.e. bone loss occurs below the neurological injury level, with all limbs affected in tetraplegia, but only the lower limbs in paraplegia[5, 17, 18]. In both paraplegics and tetraplegics, there is substantial variability in the rate of bone loss at 12 months post-injury, with some SCI patients losing bone much faster than predicted (up to 40\%), some losing bone at the predicted rate, while others lose little or no bone[19]. The rate of bone loss cannot be predicted or explained by the level of neurological injury and methods to identify those who will suffer rapid bone loss are needed.

The innovative use of statistical shape modelling (SSM) to capture bone shape has enhanced hip fracture prediction in the elderly[20-22]. SSM characterises and quantifies the natural 
shape variation of an object, overcoming the limitations of basic geometric measurements (e.g. width, length, angles) which are suitable for simpler objects. This approach has been successfully applied to study various medical conditions[20, 23-26]. Until now, bone morphology has not been investigated in SCI patients, even though it is a known determinant of bone strength, independent of BMD[27-29]. Few studies have investigated prediction of bone loss in postmenopausal women, older men or SCI patients[19, 30-33].

The aim of this study is to investigate, for the first time, the prediction of SCI related bone loss based on a single early bone scan. The specific objective of this study was to test whether femoral and tibial statistical shape models reveal shape variations that can predict the rate of bone loss in a cohort of SCI patients and act as an imaging biomarker.

\section{Material and Methods}

\subsection{Study subjects and imaging}

This study was approved by the West of Scotland Research Ethics Committee 3 in line with the declaration of Helsinki[34]. Participants gave full informed consent and their datasets were anonymised.

Images and data from a prospective study, designed to characterise bone loss in individuals with complete SCI in the first year post-injury were analysed retrospectively. Full details of the study design and findings are available elsewhere[34]. Recruitment took place between October 2008 and February 2012. In-patients at the Queen Elizabeth National Spinal Injury Unit (QENSIU), Glasgow, UK, who were no more than 5 weeks post-injury at the start of the study with a ASIA Impairment Score (AIS) of A (motor-complete, sensory-complete) or B 
(motor-complete, sensory-incomplete) according to the International Standards for Neurological Classification of Spinal Cord Injury were eligible for inclusion[35]. Patients were excluded if they had pre-SCI diagnosis of osteoporosis or medical complications associated with their injury.

Images and bone density were obtained using an XCT 3000 peripheral Quantitative Computed Tomography (pQCT) scanner (Stratec Medizintechnik GmbH). Scans were taken of the distal femur and proximal tibia at four time points. Baseline scans were taken as soon as possible after injury (all before 5 weeks post-injury) and follow up scans at 4,8 and 12 months post-injury.

Scans were taken on the 'dominant' side for all participants except when a limb on that side had a history of fractures or if the region to be scanned contained any metal components[19, 34]. All scans were taken by the same operator using the same anatomical location ( $4 \%$ total bone length) and scanning parameters as the baseline scan (slice thickness $2.4 \mathrm{~mm}$, voxel edge length $0.3 \mathrm{~mm}$ for distal femur and $0.5 \mathrm{~mm}$ for proximal tibia)[36]. Reproducibility has been previously published[36]. Images were saved as comma separated value files and converted into 8bit Tiff. The right leg was scanned for 21/25 participants. All subjects had femur BMD values within the normal range at baseline (no normative values are available for the proximal tibia)[15]. Percentage change in total BMD, trabecular BMD (calculated within the central $45 \%$ of total bone area) and Bone Mineral Content (BMC) from baseline to 12-month scan were calculated[34]. Cortical BMD was not measured as the thinness of the cortical shell at the epiphyses makes it an unreliable measure due to the partial volume effect. 


\subsection{Statistical shape modelling}

Semi-automated point placement of shape templates was performed on all baseline images using the Active Shape Model Toolkit (Visual Automation Limited, Manchester, UK). Figure 1 shows the templates for the femur (44 points) and tibia (30 points). Mode scores (output variables) were calculated by a locally developed software (Shape, Aberdeen, UK) using principal component analysis. Scores were calculated so each mode has a mean of zero and a standard deviation (SD) of 1 and is statistically independent from the other modes (Figure 2).

Intra- and inter-repeatability were assessed using the median point-to-point distance for 10 femur and tibia images each. Each image was marked up twice at least one day apart. Intraand inter-repeatability was 0.6 pixels $(0.18 \mathrm{~mm})$ and 1.2 pixels $(0.36 \mathrm{~mm})$ for the femur and 0.7 pixels $(0.35 \mathrm{~mm})$ and 1.9 pixels $(0.95 \mathrm{~mm})$ for the tibia respectively.
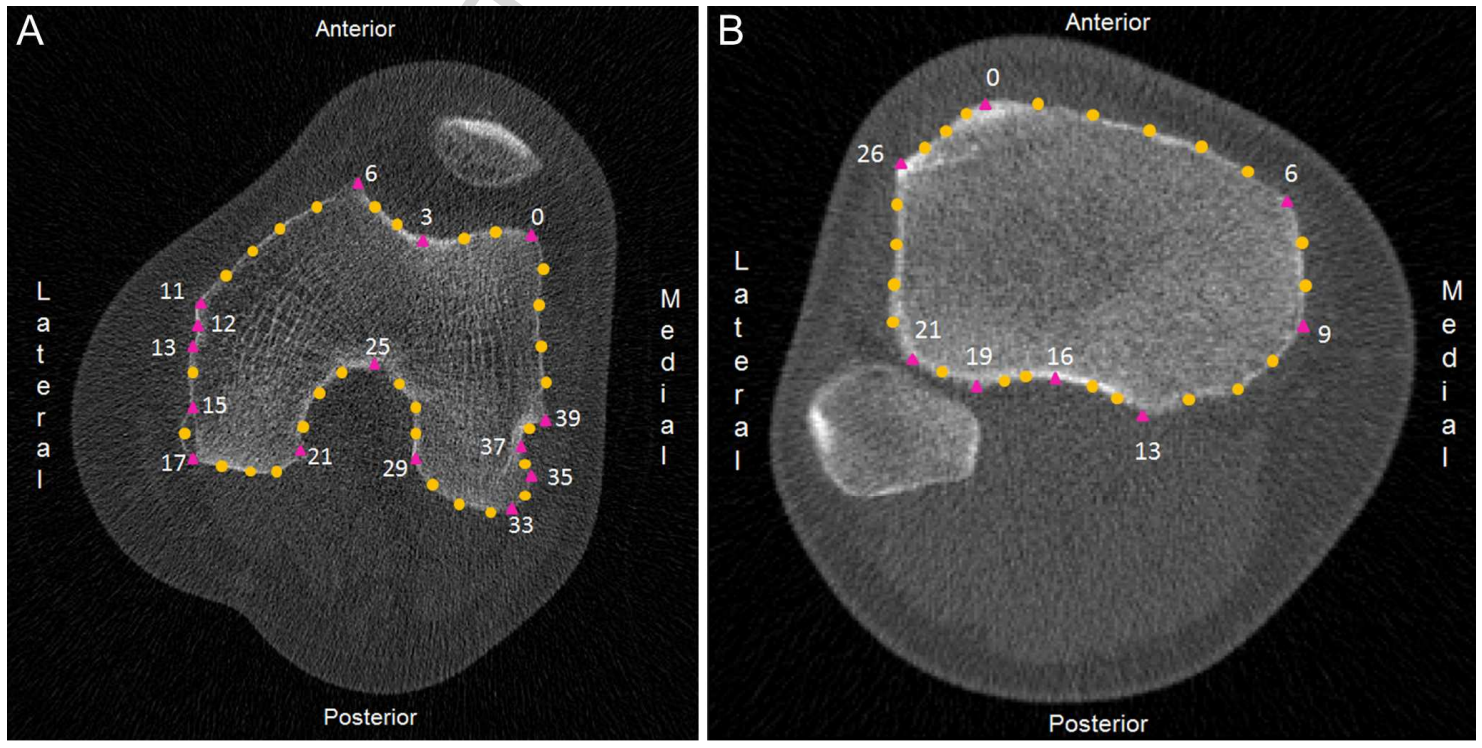

\section{Figure 1}

Femur and tibia shape models. Landmark points (triangles) mark easily identifiable anatomical or geometric features (e.g. the tip of the femoral anteromedial condyle). Nonlandmark points (circles) are equally spaced to describe the outline. 

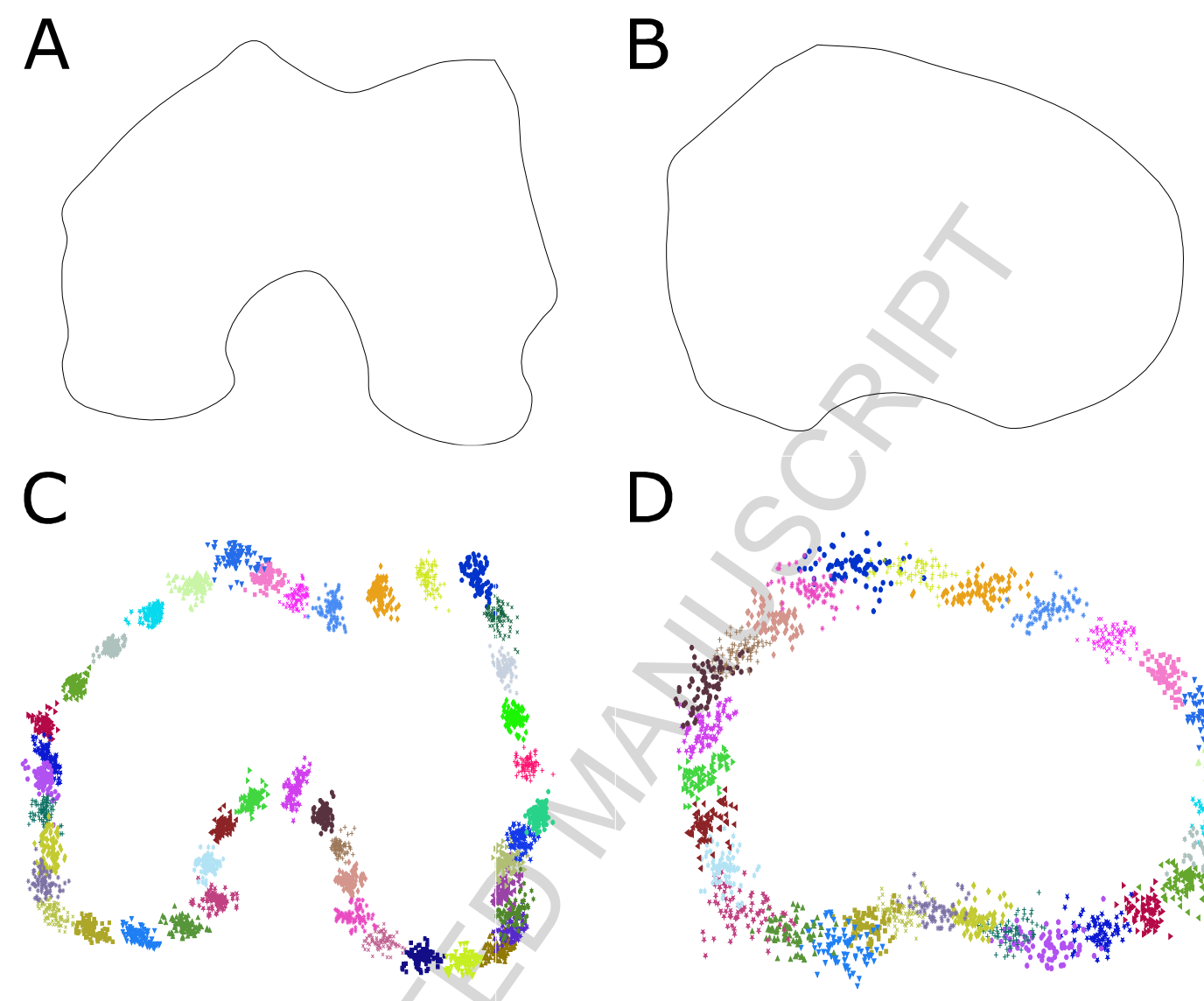

\section{Figure 2}

To calculate shape variation in the dataset, the bones must first be aligned as closely as possible by scaling, rotating and transforming the outlines (Procrustes analysis). This allows the average shape to be calculated for the femur (A) and tibia (B) and also a "point cloud" for each, (C and D respectively) showing the variation in femur and tibia points. Principal Component Analysis is then used to quantify this variation by calculating "modes" which represent different patterns of variability from the average shape. The first mode describes the largest percentage of variability in the dataset, followed by the second mode, and so on. Each image is assigned a set of mode scores (one for each mode) representing how it varies from the average shape. 


\subsection{Statistical analysis}

Statistical tests were performed using SPSS version 21.0.01 (SPSS, IBM, USA). Significance level was set at $P<0.05$. Normality testing was performed using the Shapiro-Wilk test, equality of variance using Levene's Test.

In a per-protocol analysis, subject characteristics evaluated as potential risk factors for SCI bone loss (sex, level of injury, AIS and age) were investigated using Pearson's correlation (or its non-parametric equivalent where appropriate) to test for association with 12-month BMD and BMC change. The link between baseline bone shape (mode scores from $\leq 5$ week scans) and the factors described above were assessed using t-tests and Pearson's correlation or their non-parametric equivalents. Simple and age-adjusted multiple linear regression were used to assess how much of the variance in 12-month percentage change in BMD was accounted for by baseline shape and Cohen's $f^{2}$ effect sizes calculated.

\section{Results}

\subsection{Subject demographics}

The flow chart in Figure 3 describes the study recruitment. Fifty-five eligible patients were identified and 25 were included in the study[34]. Baseline pQCT scans were available for all participants at the femur and all but 2 at the tibia. Percentage change data for BMD and BMC from baseline to 12 months were available for 19 participants at the femur and 17 at the tibia. 


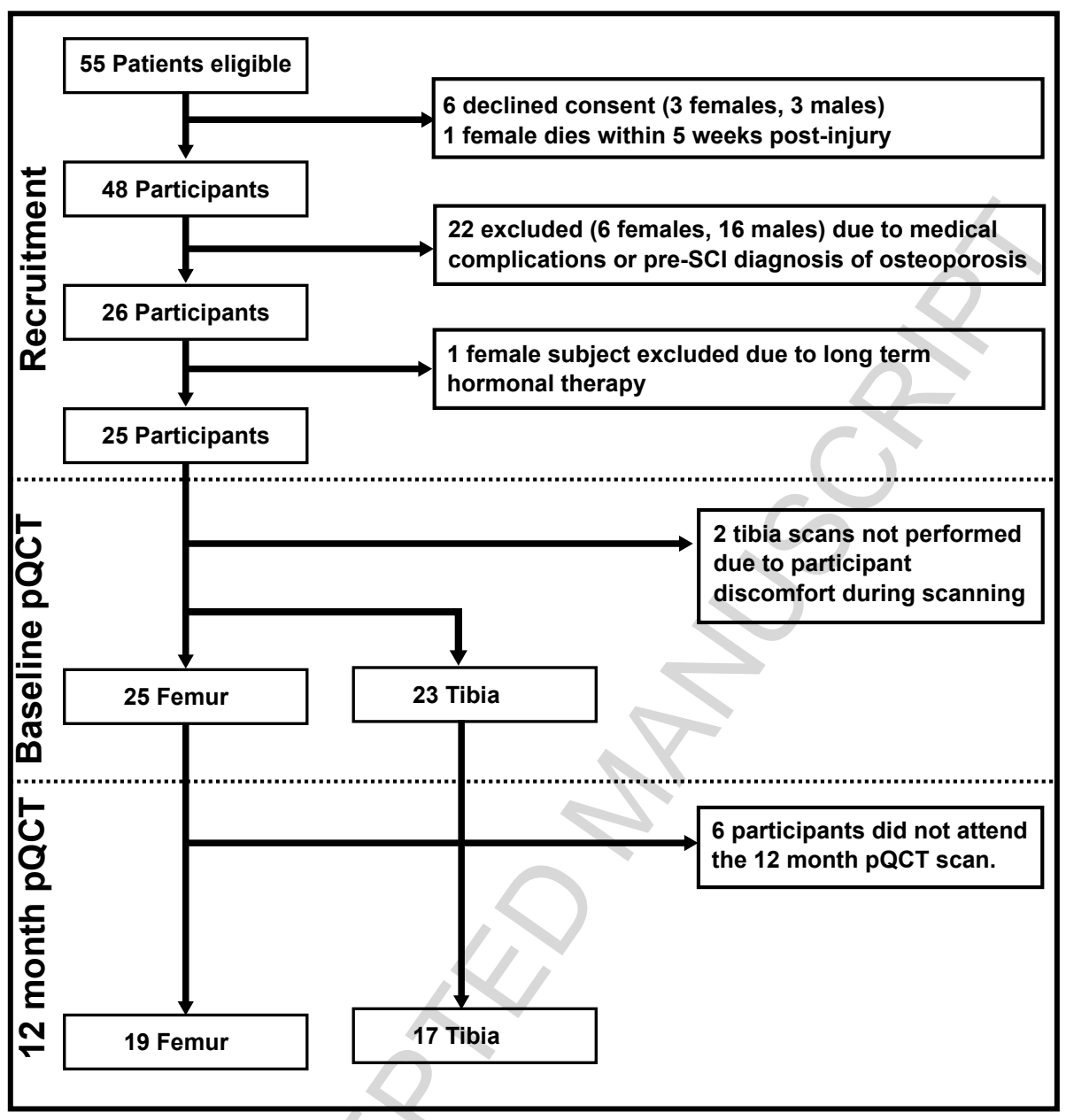

\section{Figure 3}

Flowchart showing recruitment and retention of study participants.

On average $($ Mean $\pm \mathrm{SD})$ baseline scans were performed at $1.00 \pm 0.17$ months post-injury $(n=25)$, and final scans at $12.56 \pm 0.99$ months post-injury $(n=19)$. There was no significant difference in age, gender or paraplegic/tetraplegic category between those who underwent a 12-month scan and those who did not $(P=0.220-0.675)$.

The cause of SCI in all 25 participants was trauma. There were 21 male and 4 female participants with a median age of 33.0 (Inter-quartile range=20.0-50.5) ranging from 16 to 76 years. The level of SCI ranged from cervical 4 to lumbar 1 spine and participants were 
categorised into paraplegic $(n=10)$ or tetraplegic $(n=15)$ for analysis. The majority $(n=19)$ had no motor or sensory function (AIS A), the rest $(n=6)$ had some sensory but no motor control below the injury level (AIS B).

\subsection{Baseline risk factors for SCI bone loss}

In the femur, there were no significant differences in bone loss (total BMD, trabecular BMD or BMC percentage change) when comparing sex, injury status (AIS and injury level) (Table 1) and no significant correlations with age $(P>0.05)$.

Similarly tibial total BMD, trabecular BMD and BMC change were not related to sex or AIS. However, tetraplegics had a significantly smaller 12-month total BMD loss compared with paraplegics (Table 1). Age was significantly correlated with total BMD $(r=0.66, P=0.004)$ trabecular $\mathrm{BMD}(\mathrm{r}=0.70, \mathrm{P}=0.002)$ and $\mathrm{BMC}(r=0.68, P=0.003)$ change.

Baseline height and weight data were not available due to difficulty in their measurement in the acute stage after a SCI. Spastic versus flaccid paralysis and the administration of methylprednisolone, were also not associated with 12-month BMD or BMC change (data not shown). 
Table 1: Association between baseline characteristics and 12-month percentage change in BMD and BMC at the femur and tibia.

\begin{tabular}{|c|c|c|c|c|c|c|c|}
\hline Variable & Group (N) & $\begin{array}{l}\text { Total BMD \% } \\
\text { loss }\end{array}$ & $P$ & Trabecular BMD \% loss & $P$ & BMC \% loss & $P$ \\
\hline \multicolumn{8}{|l|}{ Femur } \\
\hline Sex & $\begin{array}{l}\text { Female (4) } \\
\text { Male (15) }\end{array}$ & $\begin{array}{l}-14.5(12.0) \\
-17.9(12.2)\end{array}$ & 0.624 & $\begin{array}{l}-13.9(12.6) \\
-15.4(17.2)\end{array}$ & 0.874 & $\begin{array}{l}-15.2(14.3) \\
-18.6(12.3)\end{array}$ & 0.642 \\
\hline $\begin{array}{l}\text { Injury } \\
\text { Status }\end{array}$ & $\begin{array}{l}\text { Para (9) } \\
\text { Tetra (10) }\end{array}$ & $\begin{array}{l}-20.8(9.7) \\
-13.9(13.2)\end{array}$ & 0.217 & $\begin{array}{l}-19.2(14.3) \\
-11.5(17.4)\end{array}$ & 0.307 & $\begin{array}{l}-20.3(-32.3,-13.0) \\
-7.8(-29.6,-6.0)\end{array}$ & 0.191 \\
\hline AIS & $\begin{array}{l}\text { A (14) } \\
\text { B (5) }\end{array}$ & $\begin{array}{l}-19.3(11.4) \\
-11.2(12.5)\end{array}$ & 0.202 & $\begin{array}{l}-17.3(15.2) \\
-9.1(18.7)\end{array}$ & 0.346 & $\begin{array}{l}-19.6(13.2) \\
-13.1(9.2)\end{array}$ & 0.334 \\
\hline \multicolumn{8}{|l|}{ Tibia } \\
\hline Sex & $\begin{array}{l}\text { Female (3) } \\
\text { Male (14) }\end{array}$ & $\begin{array}{l}-28.2(17.4) \\
-25.7(14.7)\end{array}$ & 0.800 & $\begin{array}{l}-16.9(-17.6,-4.6) \\
-13.9(-48.5,-7.3)\end{array}$ & $0.900^{\dagger}$ & $\begin{array}{l}-20.9(7.4) \\
-23.0(13.7)\end{array}$ & 0.798 \\
\hline $\begin{array}{l}\text { Injury } \\
\text { Status }\end{array}$ & $\begin{array}{l}\text { Para (8) } \\
\text { Tetra (9) }\end{array}$ & $\begin{array}{l}-33.5(11.4) \\
-19.6(14.7)\end{array}$ & 0.049 & $\begin{array}{l}-29.8(21.1) \\
-12.6(15.4)\end{array}$ & 0.072 & $\begin{array}{l}-28.3(10.8) \\
-17.7(12.6)\end{array}$ & 0.083 \\
\hline AIS & $\begin{array}{l}\text { A (12) } \\
\text { B (5) }\end{array}$ & $\begin{array}{l}-24.9(-42.3,- \\
14.7)\end{array}$ & $0.527^{\dagger}$ & $\begin{array}{l}-24.1(22.6) \\
-12.5(6.7)\end{array}$ & 0.128 & $\begin{array}{l}-24.2(14.5) \\
-18.9(6.1)\end{array}$ & 0.450 \\
\hline
\end{tabular}

T-tests (presented as mean (standard deviation)) or Mann-Whitney tests (presented as median (interquartile range) and marked by $\dagger$ ) were used as appropriate. Significant results are indicated in bold. BMD $=$ Bone Mineral Density; BMC $=$ Bone Mineral Content; Para = Paraplegic; Tetra = Tetraplegic; AIS = ASIA Impairment Score 


\subsection{Femur shape}

The first 7 modes were selected for analysis from the femoral SSM. These described $74.8 \%$ of the total variance, with each mode describing at least $4.2 \%$. The remaining modes were discarded as, individually, they accounted for a small percentage of variance that was indistinguishable by eye.

\subsubsection{Femur shape and baseline characteristics}

Analysis of baseline mode scores showed that men had significantly higher mode 2 scores than women $(0.24 \pm 0.80$ and $-0.84 \pm 0.80$ respectively, $P=0.028)$ and mode 5 was both significantly higher in tetraplegics than paraplegics $(1.03 \pm 0.94$ and $-0.46 \pm 0.51$ respectively, $P=0.001)$ and significantly correlated with age $(r=0.58, P=0.009)$. There were no significant differences in mode scores between AIS A and AIS B groups for any of the modes $(P>0.05)$.

Tetraplegics were significantly older than paraplegics, mean age $=46.6 \pm 20.0$ and $29.6 \pm 11.5$ years respectively $(P=0.039)$, which may explain why both tetraplegic and older subjects had higher baseline mode 5 scores.

\subsubsection{Femur shape and bone loss}

Only mode 4 (Figure 4A) correlated with 12-month percentage changes in total or trabecular BMD or BMC ( $r=-0.53, P=0.019$ (Pearson's correlation), $\mathrm{r}=-0.51 \mathrm{P}=0.026$ and $r=-0.48$, 
$P=0.038$ (Spearman's correlation)) respectively i.e. those who lost a higher percentage of BMD and BMC at 12 months were more likely to have a higher mode 4 score at baseline.

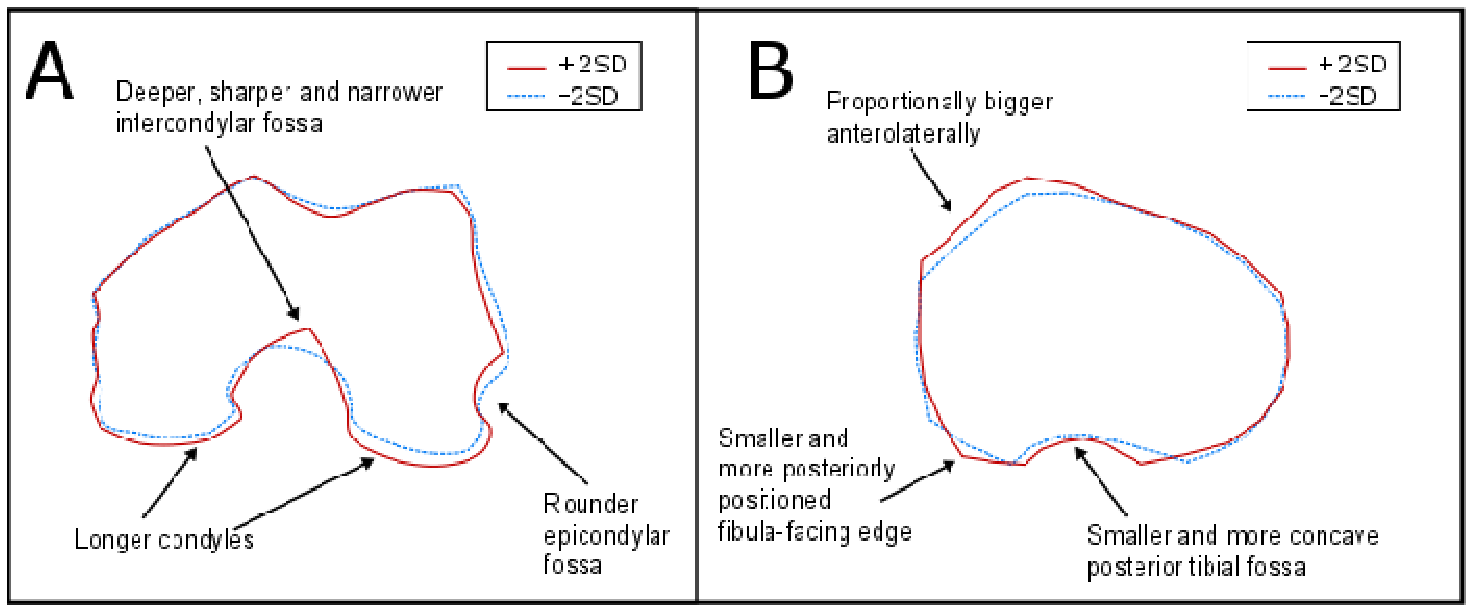

\section{Figure 4}

(A) Femur mode 4. Higher mode 4 scores show longer condyles, a more concave epicondylar fossa and a deeper, sharper and narrower intercondylar fossa, compared to lower scores. (B) Tibia mode 3 . Higher mode 3 scores show a more concave posterior tibial fossa and are proportionally bigger anterolaterally, compared to lower scores. SD = Standard Deviation.

Using multiple linear regression, mode 4 accounted for $28.5 \%$ of variance in total BMD change $\left(R^{2}=0.29, P=0.019\right)$. Each 1 SD increase in mode 4 was associated with a loss of an additional $6.1 \%$ total BMD $(95 \% \mathrm{CI}-11.0,-1.2)$ constant $=-17.9 \% \mathrm{f}^{2}=0.41$. This result was still significant after adjustment for age with 6.4\% additional total BMD loss (95\%CI -11.3, 1.5) per SD increase $\left(R^{2}=0.39\right.$, constant $\left.=-26.3 \%, P=0.014, \mathrm{f}^{2}=0.64\right)$. No other modes were significant before or after adjustment for age $(P>0.05)$.

For trabecular BMD change, mode 4 accounted for $26 \%$ of variance $\left(\mathrm{R}^{2}=0.26, \mathrm{P}=0.026\right)$. Each 1SD increase was associated with a loss of an additional $8.1 \%$ trabecular BMD (95\%CI 
$-15.1,-1.1)$ constant $=-16.5 \%, \mathrm{f}^{2}=0.35$. This result was still significant after adjustment for age with $8.2 \%$ additional BMD loss $(95 \% \mathrm{CI}-14.9,-1.5)$ per SD increase $\left(\mathrm{R}^{2}=0.37\right.$, constant $=-$ $\left.27.8 \%, \mathrm{P}=0.019, \mathrm{f}^{2}=0.59\right)$.

\subsection{Tibia shape}

The first 7 modes accounted for $87.1 \%$ of the total variance, with each accounting for at least $2.8 \%$. As previously, the remaining modes were discarded.

\subsubsection{Tibia shape and baseline characteristics}

No significant differences were observed in any of the mode scores between males and females, para- and tetraplegic groups or between AIS A and B groups $(P>0.05)$ and there were no correlation between mode scores and age $(P>0.05)$. However, when examining all baseline data $(n=23)$, a significant negative correlation was found between mode 3 and age, so older subjects were more likely to have a high mode 3 score $(r=-0.42, P=0.044)$.

\subsubsection{Tibia shape and bone loss}

A significant negative correlation was found between mode 3 (Figure 4B) and percentage total BMD ( $r=-0.49, P=0.048)$ and BMC change $(r=-0.52, P=0.032)$ there was a borderline negative correlation between mode 3 and trabecular BMD change ( $r=-0.48, P=0.054)$. There was a significant negative correlation between mode 1 and trabecular BMD change $(r=-0.55$, $\mathrm{P}=0.022)$ whilst $\mathrm{BMC}$ change was borderline significant $(r=-0.48, P=0.053)$. 
Multiple linear regression found that mode 3 accounted for $24 \%$ of variability in tibial total BMD change before adjustment for age, $\left(R^{2}=0.24\right)$ with $7.0 \%$ more total BMD loss $(95 \% \mathrm{CI}$ 13.9,-0.06) for every $1 \mathrm{SD}$ increase (constant $=-25.3 \%, P=0.048, \mathrm{f}^{2}=0.31$ ) which became nonsignificant after adjustment for age $\left(\mathrm{R}^{2}=0.48\right.$, with $4.3 \%$ more total BMD loss (95\%CI -10.7, $2.1 \%$ ) for each $1 \mathrm{SD}$ increase, constant=-41.0, $\left.P=0.169, \mathrm{f}^{2}=0.92\right)$.

Trabecular bone loss was also predicted by mode 3, which accounted for $42 \%$ of variance in trabecular BMD change $\left(R^{2}=0.42, P=0.005\right)$. Each $1 \mathrm{SD}$ increase in mode 3 was associated with a loss of an additional 12.6\% trabecular BMD (95\%CI -20.7, -4.4) constant=-19.1\%, $\mathrm{f}^{2}=0.72$. This result was still significant after adjustment for age with $9.4 \%$ additional BMD loss $(95 \% \mathrm{CI}-16.9,-1.9)$ per SD increase $\left(R^{2}=0.61\right.$, constant $\left.=-37.7 \%, P=0.018, \mathrm{f}^{2}=1.53\right)$.

\section{Discussion}

This study found that bone shape is a repeatable early predictor of bone loss after a SCI, even after adjustment for age, the strongest baseline predictor (since the young showed a faster rate of bone loss). The predictive capacity of shape was stronger for trabecular than total bone loss in both the femur and tibia. In the femur, an elongated intercondylar femoral notch represented by a $1 \mathrm{SD}$ higher score for femur-mode 4 at $<5$ weeks corresponded to an extra 8.2\% femoral trabecular BMD loss at 12 months post-injury. Similarly, at the tibia, a more concave posterior tibial fossa and a proportionally bigger anterolateral section, represented by a 1SD higher baseline for tibia-mode 3 corresponded to an extra $9.4 \%$ loss of tibial trabecular BMD at 12 months. 
Visualisation of the modes allows description of the shapes most predictive of bone loss. In the femur, a higher mode 4 (associated with increased bone loss) had longer condyles, a more concave epicondylar fossa and a deeper, sharper and narrower intercondylar notch compared with lower scores (Figure 4A). The tibial shape associated with a higher mode 3 score had a more concave posterior tibial fossa and is proportionally bigger anterolaterally (Figure 4B), compared with the lower mode 3 scores, which were associated with a reduced tibial bone loss.

In both the femur and the tibia, the most striking shape differences were observed around muscle and ligament insertion points. These results are perhaps not unexpected since these biomechanically active regions are vital for maintaining skeletal tissue through transmission of loading forces. It is unlikely that the shape of the femur or tibia would have changed significantly from when the injury first happened to the time of the first scan, however we cannot be certain of this. The observed difference in shape may be congenital and put patients at a higher risk of bone loss due to the effects of muscle on bone or vice versa.

Alone, the shape models explained between $24 \%$ and $42 \%$ of the subsequent bone loss. Including age in the regression increased this substantially (between $37 \%$ and $61 \%$ ), with the highest percentage in the trabecular bone of the tibia. Most effect sizes were classed as "large" using Cohen's definitions [37], with just one classed as "medium" $(<0.35)$. Whilst it is always recommended to use descriptors such as "large", "medium" and "small" with caution, even by Cohen himself, these findings indicate our models may have clinical, not just statistical significance. 
There are no published studies that have addressed prediction of the speed of disuse related bone loss in SCI, and very few in post-menopausal osteoporosis or older men[30-33]. In postmenopausal and SCI osteoporosis the primary focus has been on predicting fractures. SSM has been previously used to predict hip fractures in post-menopausal women using radiographs or Dual Energy X-ray Absorptiometry (DXA) scans of the proximal femur[2022]. The present study is the first to use SSM to study SCI-induced osteoporosis and moreover it is the first to use SSM to predict bone loss.

Previous studies have made important advances in our understanding of SCI bone loss by describing it using DXA, pQCT, as well as blood and urine concentrations of bone turnover markers[12, 14-17, 38-44]. There is potential for data from both longitudinal and crosssectional studies in this field to be assessed for association with rate of bone loss. Such information could add to the predictive ability of the findings from the current study.

Since the findings of this study are novel, there are no directly comparable studies. The few studies that have assessed distal femur or proximal tibia shape have assessed it in the context of knee osteoarthritis or clinical studies looking to predict outcome of interventions based on bone morphology. Interestingly all these studies noted differences in the femoral intercondylar notch shape associated with either osteoarthritis or the success of cruciate ligament reconstruction, and many proposed that this may arise due to differences in cruciate ligament attachment[45-48]. Differences in the shape of the distal femur based on age and sex have also been reported elsewhere[46].

In the general population, a decrease in BMD is typically associated with ageing. However in this study, older participants had a slower rate of loss at the proximal tibia. This may be 
because they are likely to be less physically active and may have already suffered from some generalised loss of muscle strength, and so are not as reliant on this for BMD maintenance[49].

Key strengths of this study are the longitudinal design, starting as soon as practically possible after injury, the use of clinically relevant skeletal sites for the SCI population (femur and tibia)[34], and the high repeatability of both the pQCT measurements,[36] and the shape models (inter-repeatability for both the femur and tibia $<1 \mathrm{~mm}$ ). This study is one of the largest longitudinal studies of acute SCI. There are around 100 cases of SCI per year in Scotland and approximately half of these are motor complete injuries, which was the focus of this study.

The patient sample was representative of the SCI population in terms of sex, average age and age range[50,51], but there were limitations. Although the majority of the eligible patients admitted to QENSIU gave consent to participate, many could not be scanned at less than five weeks post injury due to medical complications. These patients were not included in the study and the participants may therefore have been a healthier group.

Other than age, the shape models developed in this study are the first biomarkers of bone loss identified in the SCI population. They have the potential to be used as a tool to help identify at risk SCI patients so that they can be monitored or treated at an early stage.

\section{Conclusion}

In conclusion bone shape measurement within one month of injury is a predictor of SCI bone loss at the distal femur and the proximal tibia, one year post-injury. This imaging biomarker 
may help in the early detection, treatment and prevention of SCI osteoporosis, with the potential to reduce the incidence of fractures and their associated morbidity.

\section{Acknowledgments}

We would like to thank the study participants and the clinical and research staff at the Queen Elizabeth National Spinal Injury Unit, as without them this study would not have been possible. We are grateful for the funding received from Glasgow Research Partnership in Engineering for the employment of SC during data collection for this study. We would like to thank the Royal Society of Edinburgh's Scottish Crucible scheme for providing the opportunity for this collaboration to occur. We are also indebted to Maria Dumitrascuta for her time and effort in producing inter-repeatability results for the shape models. 


\section{Reference List}

1 International Campaign for Cures of spinal cord injury Paralysis. http://www campaignforcure org/ 2009 July;(Accessed 16 Feb 2015).

2 Maimoun L, Fattal C, Micallef JP, et al. Bone loss in spinal cord-injured patients: from physiopathology to therapy. Spinal Cord 2006;44:203-10.

3 Jiang SD, Dai LY, Jiang LS. Osteoporosis after spinal cord injury. Osteoporos Int 2006;17:180-92.

4 Fattal C, Mariano-Goulart D, Thomas E, et al. Osteoporosis in Persons With Spinal Cord Injury: The Need for a Targeted Therapeutic Education. Arch Phys Med Rehabil 2011;92:59-67.

5 Vestergaard P, Krogh K, Rejnmark L, et al. Fracture rates and risk factors for fractures in patients with spinal cord injury. Spinal Cord 1998;36:790-6.

6 Carbone LD, Chin AS, Burns SP, et al. Morbidity following lower extremity fractures in men with spinal cord injury. Osteoporos Int 2013;24:2261-7.

7 Charmetant C, Phaner V, Condemine A, et al. Diagnosis and treatment of osteoporosis in spinal cord injury patients: A literature review. Ann Phys Rehabil Med 2010;53:65568.

8 National Institute for Health and Care Excellence. Alendronate, etidronate, risedronate, raloxifene, strontium ranelate and teriparatide for the secondary prevention of osteoporotic fragility fractures in postmenopausal women (amended). London: National 
Institute for Health and Care Excellence Technology Appraisal Guidance; 2008 Oct. Report No.: ta161.

9 National Institute for Health and Care Excellence. Osteoporosis: assessing the risk of fragility fracture. London: National Institute for Health and Care Excellence Guidlines; 2012 Aug. Report No.: CG146.

10 Kanis JA, Johnell O, Oden A, et al. FRAX (TM) and the assessment of fracture probability in men and women from the UK. Osteoporos Int 2008;19:385-97.

11 Bubbear JS, Gall A, Middleton FRI, et al. Early treatment with zoledronic acid prevents bone loss at the hip following acute spinal cord injury. Osteoporos Int 2011;22:271-9.

12 Dudley-Javoroski S, Shields RK. Regional cortical and trabecular bone loss after spinal cord injury. J Rehabil Res Dev 2012;49:1365-76.

13 Frotzler A, Coupaud S, Perret C, et al. High-volume FES-cycling partially reverses bone loss in people with chronic spinal cord injury. Bone 2008;43:169-76.

14 Biering-Sorensen F, Bohr HH, Schaadt OP. Longitudinal-Study of Bone-Mineral Content in the Lumbar Spine, the Forearm and the Lower-Extremities After SpinalCord Injury. European Journal of Clinical Investigation 1990;20:330-5.

15 Eser P, Frotzler A, Zehnder Y, et al. Relationship between the duration of paralysis and bone structure: a pQCT study of spinal cord injured individuals. Bone 2004;34:869-80.

16 Frotzler A, Berger M, Knecht H, et al. Bone steady-state is established at reduced bone strength after spinal cord injury: A longitudinal study using peripheral quantitative computed tomography (pQCT). Bone 2008;43:549-55. 
17 Frey-Rindova P, de Bruin ED, Stussi E, et al. Bone mineral density in upper and lower extremities during 12 months after spinal cord injury measured by peripheral quantitative computed tomography. Spinal Cord 2000;38:26-32.

18 Gifre L, Vidal J, Carrasco J, et al. Incidence of skeletal fractures after traumatic spinal cord injury: a 10-year follow-up study. Clin Rehabil 2014;28:361-9.

19 Coupaud S, Mclean AN, Lloyd S, et al. Predicting patient-specific rates of bone loss at fracture-prone sites after spinal cord injury. Disabil Rehabil 2012;34:2242-50.

20 Goodyear SR, Barr RJ, McCloskey E, et al. Can we improve the prediction of hip fracture by assessing bone structure using shape and appearance modelling? Bone 2013;53:188-93.

21 Gregory JS, Testi D, Stewart A, et al. A method for assessment of the shape of the proximal femur and its relationship to osteoporotic hip fracture. Osteoporos Int 2004;15:5-11.

22 Baker-LePain JC, Luker KR, Lynch JA, et al. Active Shape Modeling of the Hip in the Prediction of Incident Hip Fracture. J Bone Miner Res 2011;26:468-74.

23 Miller NA, Gregory JS, Aspden RM, et al. Using Active Shape Modeling Based on MRI to Study Morphologic and Pitch-Related Functional Changes Affecting Vocal Structures and the Airway. J Voice28:554-64.

24 Pavlova A, Meakin J, Cooper K, et al. The lumbar spine has an intrinsic shape specific to each individual that remains a characteristic throughout flexion and extension. Eur Spine J 2014;23:26-32. 
25 Cootes TF, Hill A, Taylor CJ, et al. Use of active shape models for locating structures in medical images. Image Vis Comput 1994;12:355-65.

26 Barr RJ, Gregory JS, Reid DM, et al. Predicting OA progression to total hip replacement: can we do better than risk factors alone using active shape modelling as an imaging biomarker? Rheumatology (Oxford) 2012;51:562-70.

27 Bruno AG, Broe KE, Zhang X, et al. Vertebral size, bone density, and strength in men and women matched for age and areal spine BMD. 2014;29:562-9.

28 Bouxsein ML. Overview of Bone Structure and Strength. Genetics of Bone Biology and Skeletal Disease. 2013:25-34.

29 Bass SL, Saxon L, Daly RM, et al. The effect of mechanical loading on the size and shape of bone in pre-, peri-, and postpubertal girls: A study in tennis players. $J$ Bone Miner Res 2002;17:2274-80.

30 Christiansen C, Riis BJ, Rodbro P. Prediction of Rapid Bone Loss in Postmenopausal Women. Lancet 1987;1:1106-8.

31 Vestergaard P. Prediction of changes in bone mineral in early postmenopausal women Aarhus University Hospital; 2000.

32 Curtis JR, Ewing SK, Bauer DC, et al. Association of intact parathyroid hormone levels with subsequent hip BMD loss: the Osteoporotic Fractures in Men (MrOS) Study. $J$ Clin Endocrinol Metab 2012;97:1937-44.

33 Cawthon PM, Ewing SK, Mackey DC, et al. Change in hip bone mineral density and risk of subsequent fractures in older men. J Bone Miner Res 2012;27:2179-88. 
34 Coupaud S, Mclean AN, Purcell M, et al. Decreases in bone mineral density at cortical and trabecular sites in the tibia and femur during the first year of spinal cord injury. Bone 2015;74C:69-75.

35 Kirshblum SC, Burns SP, Biering-Sorensen F, et al. International standards for neurological classification of spinal cord injury (Revised 2011). 2011;34:535-46.

36 Findlay CM. Image analysis tool for the characterisation of bone turnover in the appendicular skeleton University of Glasgow; 2012.

37 Cohen J. Statistical Power Analysis for the Behavioral Sciences. 2 ed. Hillsdale, New Jersey: Lawrence Erlbaum Associates 1988.

38 Dauty M, Verbe BP, Maugars Y, et al. Supralesional and sublesional bone mineral density in spinal cord-injured patients. Bone 2000;27:305-9.

39 Doherty AL, Battaglino RA, Donovan J, et al. Adiponectin Is a Candidate Biomarker of Lower Extremity Bone Density in Men With Chronic Spinal Cord Injury. J Bone Miner Res 2014;29:251-9.

40 Edwards WB, Schnitzer TJ, Troy KL. Bone mineral and stiffness loss at the distal femur and proximal tibia in acute spinal cord injury. Osteoporos Int 2014;25:1005-15.

41 Maimoun L, Couret I, Micallef JP, et al. Use of bone biochemical markers with dualenergy X-ray absorptiometry for early determination of bone loss in persons with spinal cord injury. Metabolism 2002;51:958-63.

42 Morse LR, Sudhakar S, Lazzari AA, et al. Sclerostin: a candidate biomarker of SCIinduced osteoporosis. Osteoporos Int 2013;24:961-8. 
43 Roberts D, Lee W, Cuneo RC, et al. Longitudinal study of bone turnover after acute spinal cord injury. J Clin Endocrinol Metab 1998;83:415-22.

44 Wilmet E, Ismail AA, Heilporn A, et al. Longitudinal-Study of the Bone-Mineral Content and of Soft-Tissue Composition After Spinal-Cord Section. Paraplegia $1995 ; 33: 674-7$.

45 Shepstone L, Rogers J, Kirwan JR, et al. Shape of the intercondylar notch of the human femur: a comparison of osteoarthritic and non-osteoarthritic bones from a skeletal sample. Ann Rheum Dis 2001;60:968-73.

46 Stevens SD, Vidarsdottir US. Morphological changes in the shape of the nonpathological bony knee joint with age: A morphometric analysis of the distal femur and proximal tibia in three populations of known age at death. International Journal of Osteoarchaeology 2008; 18:352-71.

47 Eggerding V, van Kuijk KS, van Meer BL, et al. Knee shape might predict clinical outcome after an anterior cruciate ligament rupture. Bone Joint J 2014;96-B:737-42.

48 Neogi T, Bowes MA, Niu J, et al. Magnetic resonance imaging-based threedimensional bone shape of the knee predicts onset of knee osteoarthritis: data from the osteoarthritis initiative. Arthritis Rheum 2013;65:2048-58.

49 Kaptoge S, Dalzell N, Jakes RW, et al. Hip section modulus, a measure of bending resistance, is more strongly related to reported physical activity than BMD. Osteoporos Int 2003;14:941-9.

50 McDonald JW, Sadowsky C. Spinal-cord injury. Lancet 2002;359:417-25. 
51 Hagen EM, Rekand T, Gilhus NE, et al. Traumatic spinal cord injuries-incidence, mechanisms and course. Tidsskr Nor Laegeforen 2012;132:831-7.

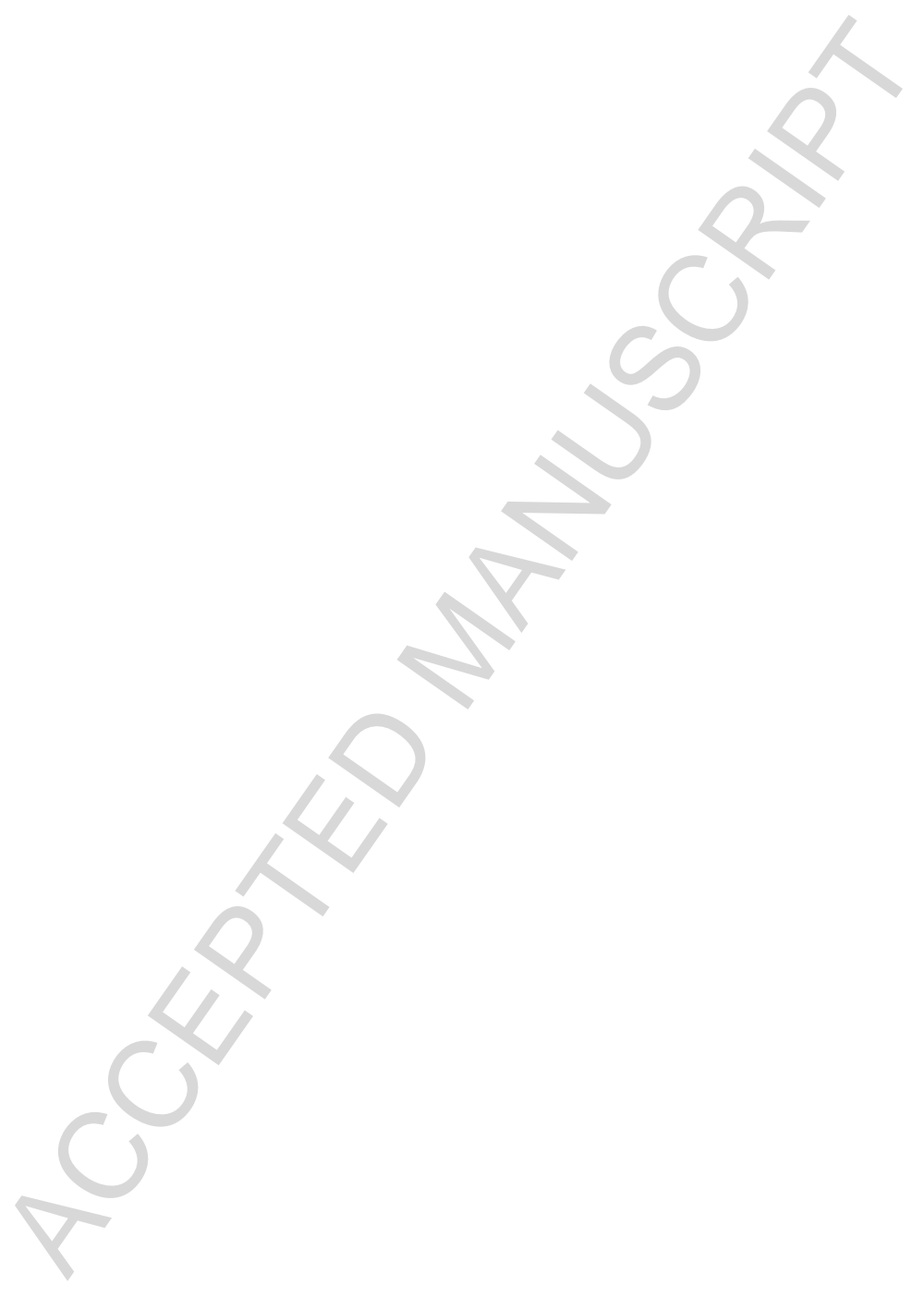




\section{Highlights}

- Links between bone shape and bone loss following spinal cord injury were explored

- Images and BMD were taken from pQCT of the distal femur and proximal tibia

- Tibial trabecular BMD loss at $1 \mathrm{yr}$ was predicted by a concave posterior tibial fossa

- An elongated intercondylar femoral notch predicted femoral trabecular BMD loss

- Baseline bone shape predicted 12-month bone loss in spinal cord injury patients 